\title{
Interspecies transmission to bovinized transgenic mice uncovers new features of a CH1641-like scrapie isolate
}

\author{
Kohtaro Miyazawa ${ }^{1 *} \mathbb{0}$, Kentaro Masujin², Yuichi Matsuura', Yoshifumi Iwamaru', Takashi Yokoyama³ \\ and Hiroyuki Okada'
}

\begin{abstract}
In animal prion diseases, including bovine spongiform encephalopathy (BSE) in cattle, chronic wasting disease in cervids, and scrapie in sheep and goats, a disease-associated isoform of prion protein ( $\left(\mathrm{Pr}^{\mathrm{d}}\right)$ accumulates in the brains of affected animals. Although the CH1641 scrapie isolate was experimentally established in the UK, a few natural $\mathrm{CH} 1641$-like scrapie cases have been reported in France and the UK. The molecular mass of the unglycosylated protease-resistant core of $\mathrm{PrP}^{\mathrm{d}}$ (PrPres) is known to be similar between $\mathrm{CH} 1641$-like scrapie and experimental BSE in sheep. We previously established an experimental CH1641-like scrapie isolate (Sh294) from a natural classical scrapie case. Here, we demonstrated that the Sh294 isolate was independent of both classical and atypical BSEs by crossspecies transmission to bovine PrP overexpressing (TgBoPrP) mice and wild-type mice. Interestingly, we found that the Sh294 isolate altered its host range by the transmission to TgBoPrP mice, and we succeeded in the first stable reproduction of $\mathrm{CH} 1641$-like scrapie specific PrPres banding patterns with the $\sim 12-\mathrm{kDa}$ small C-terminal fragment in wild-type mice. This study provides new insight into the relationship between CH1641-like scrapie isolates and BSEs. In addition, interspecies transmission models such as we have demonstrated here could be a great help to investigate the origin and host range of animal prions.
\end{abstract}

\section{Introduction}

Transmissible spongiform encephalopathies (TSEs), or prion diseases, are fatal neurodegenerative disorders that affect both humans and animals [1]. Prion diseases are characterized by spongiform changes and accumulation of a disease-associated isoform of prion protein $\left(\operatorname{PrP}^{\mathrm{d}}\right)$, which is generated by post-transcriptional modification of host cellular prion protein $\left(\operatorname{PrP}^{\mathrm{C}}\right)$, in the brains of affected hosts [2]. $\mathrm{PrP}^{\mathrm{d}}$ (or prion) is believed to be a causative agent of TSEs [3]. Bovine spongiform encephalopathy (BSE) is a prion disease in cattle [4] that was first identified in the UK and spread to European as well as North American countries and Japan through feeding of BSE-contaminated meat and bone meal [5]. This

\footnotetext{
*Correspondence: miyazawak@affrc.go.jp

1 Prion Disease Unit, Division of Transboundary Animal Disease, National Institute of Animal Health (NIAH), National Agriculture and Food Research Organization (NARO), Tsukuba, Ibaraki, Japan

Full list of author information is available at the end of the article
}

epidemic BSE is now called classical (C-) BSE because two other atypical disease phenotypes (H-BSE and L-BSE) have been reported. Based on the molecular weight of the proteinase K-resistant core of $\operatorname{PrP}^{\mathrm{d}}$ (PrPres) determined by Western blot (WB) analysis, H-BSE exhibits higher-molecular-weight PrPres banding patterns than C-BSE does [6], whereas L-BSE shows a lowermolecular-weight banding pattern [7]. Despite extensive studies, the origin of BSEs remains unknown.

Scrapie is a prion disease in sheep and goats that has been suspected to be the origin of BSEs since the discovery of the first C-BSE case in 1986. Scrapie is also classified into two disease phenotypes, termed classical and atypical scrapie [8]. To test the scrapie origin hypothesis, several experimental transmissions of classical scrapie isolates to cattle have been performed in the USA and UK [9-13]. However, the biochemical and pathological properties of accumulated $\operatorname{PrP}^{\mathrm{d}}$ in the brains of cattle 
infected with classical scrapie isolates are not consistent with those of cattle infected with C-BSE.

The CH1641 scrapie isolate was experimentally established from a case of natural classical scrapie that occurred in the UK [14]. In contrast to classical scrapie isolates, this isolate is biochemically characterized by a lower molecular mass of the unglycosylated PrPres compared with that of classical scrapie isolates and an additional $\sim 12-\mathrm{kDa}$ small C-terminal PrPres fragment [15, 16]. To date, a few natural $\mathrm{CH} 1641$-like scrapie cases have been reported in France and the UK $[17,18]$. However, no transmission studies of $\mathrm{CH} 1641$ or $\mathrm{CH} 1641$-like scrapie isolates to cattle have been reported. In our previous work, we experimentally established a CH1641-like scrapie isolate (Sh294) from a case of natural classical scrapie that occurred in the USA [19]. Sh294 cannot transmit to wild-type mice, similar to the British CH1641 scrapie isolate, but can transmit to bovine PrP-overexpressing (TgBoPrP) mice [14, 19]. Interestingly, these mice showed clinical signs of the disease similar to those of L-BSE infection.

In this study, we aimed to determine the relationship between CH1641-like scrapie and BSEs (particularly L-BSE) in cattle by further investigating the biological and biochemical properties of the Sh294 isolate in TgBoPrP mice. Because we have reported that L-BSE acquired transmissibility to wild-type mice after passage in sheep [20], we next attempted to transmit the Sh294 isolate passaged in TgBoPrP mice (designated as TgBo-passaged Sh294) to wild-type mice to examine the effects of interspecies transmission on the host range of the CH1641like scrapie isolate.

\section{Materials and methods}

\section{Mouse inoculation}

TgBoPrP mice were intracerebrally inoculated with $20 \mu \mathrm{L}$ of $10 \%$ (weight/volume) brain stem homogenates from a Corriedale sheep with experimentally developed CH1641-like scrapie (Sh294) [19]. To compare the biological and biochemical properties of this $\mathrm{CH} 1641$ like scrapie isolate with those of three different BSE strains, TgBoPrP mice were also intracerebrally inoculated with $20 \mu \mathrm{L}$ of $10 \%$ brain stem homogenates from cattle experimentally affected with L-, C-, and H-BSE. PrPres-positive mouse brains were used for subsequent passages in TgBoPrP mice. TgOvPrP59 mice, which overexpress ovine $\operatorname{PrP}_{\mathrm{ARQ}}$ (A136, R154, Q171) under the control of the neuron-specific enolase promoter [21] (a kind gift from Dr. Thierry Baron, Agence Nationale de Sécurité Sanitaire, Lyon, France) were also used for the intracerebral inoculation of the Sh294 isolate. To test the cross-species transmission ability of the TgBo-passaged Sh294 isolate, further transmission studies to wild-type mice were conducted. Threeweek-old outbred ICR mice (SLC, Shizuoka, Japan) were intracerebrally inoculated with $20 \mu \mathrm{L}$ of $10 \%$ brain homogenates from TgBoPrP mice infected with Sh294. To confirm the adaptation of the TgBo-passaged Sh294 isolate in ICR mice, subsequent passages were conducted. Additionally, subsequent passages of sheep-passaged L-BSE [22] in ICR mice were also conducted to compare with TgBo-passaged Sh294 in ICR mice. Mouse-adapted C-BSE (mBSE) and three laboratory scrapie strains (ME7, 22L, and Chandler) passaged in ICR mice were also used in this study. Mice were monitored daily for the presence of clinical signs, such as rough fur, hunched posture, and emaciation. All challenged mice were sacrificed under sevoflurane anesthesia when they began to exhibit signs of distress or progression of the disease was evident. The survival periods in this study were determined as the time elapsed from inoculation to the clinical endpoint or sudden death of mice that were pathologically and/or biochemically positive for $\operatorname{PrP}^{\mathrm{d}}$.

\section{Antibodies used in this study}

Information on monoclonal anti-PrP antibodies used in this study is detailed in Table 1. Polyclonal rabbit antiglial fibrillary acidic protein (GFAP) antibodies (Dako, Carpinteria, CA, USA) were also used for the immunostaining of astrocytes.

Table 1 Monoclonal anti-PrP antibodies used in this study

\begin{tabular}{llll}
\hline Clone & $\begin{array}{l}\text { Epitope location } \\
\text { on bovine PrP }\end{array}$ & Species reactivity & Vendor \\
\hline P4 & $101-107$ & Cattle, sheep & R-Biopharm, Darmstadt, Germany \\
$6 H 4$ & $156-163$ & Cattle, sheep, mice & Prionics, Schlieren, Switzerland \\
SAF84 & $175-180$ & Cattle, sheep, mice & Bertin Pharma, Montigny le Bretonneux, France \\
31 C6 & $155-163$ & Cattle, sheep, mice & Frontier Science, Hokkaido, Japan \\
T1 & $149-155$ & Cattle, sheep, mice & Not for sale (See reference number 41.) \\
\hline
\end{tabular}




\section{Histopathology and immunohistochemistry}

The left hemispheres of mouse brains were fixed in $10 \%$ buffered formalin containing $10 \%$ methanol. Formalinfixed brains were immersed in $98 \%$ formic acid for $60 \mathrm{~min}$ to reduce infectivity, embedded in paraffin, and sectioned for histological evaluation by staining with hematoxylin and eosin (H\&E). For PrP ${ }^{\mathrm{d}}$ immunohistochemistry, 31C6 [23] and T1 [24] monoclonal antibodies (mAbs) were used. After appropriate epitope retrieval with hydrate autoclaving, brain sections were incubated with primary antibodies. After washing, the sections were incubated with an anti-mouse, universal horseradish peroxidase (HRP)-conjugated polymer (Nichirei Histofine Simple Stain MAX-PO (M); Nichirei Biosciences Inc., Tokyo, Japan), and $\operatorname{PrP}^{\mathrm{d}}$ was visualized with 3,3'-diaminobenzidine tetrachloride as the chromogen. Finally, the sections were lightly counterstained with Mayer's hematoxylin. For dual immunofluorescence, the sections were incubated for $60 \mathrm{~min}$ with $\mathrm{T} 1 \mathrm{mAbs}$ and polyclonal rabbit anti-GFAP antibodies. After washing, the sections were incubated with Alexa Fluor 488-conjugated goat antimouse IgG (1:450; Molecular Probes, Portland, OR, USA) for $\operatorname{PrP}^{\mathrm{d}}$ and Alexa Fluor 546-conjugated goat anti-rabbit IgG (1:450; Molecular Probes) for GFAP. Finally, the sections were counterstained with Topro-3 (1:2000; Fisher Scientific K.K., Yokohama, Japan). Immunofluorescence was evaluated using a Zeiss LSM 510 laser scanning confocal microscope (Carl Zeiss, Oberkochen, Germany).

\section{PrPres detection by WB}

Brain homogenates (20\%) were mixed with an equal volume of detergent buffer containing $4 \%(\mathrm{w} / \mathrm{v})$ Zwittergent 3-14 (Merck Japan, Tokyo, Japan), 1\% (w/v) Sarkosyl (Sigma-Aldrich Japan, Tokyo, Japan), $100 \mathrm{mM} \mathrm{NaCl}$, and $50 \mathrm{mM}$ Tris- $\mathrm{HCl}$ (pH 7.6), and incubated for $30 \mathrm{~min}$ with collagenase (Wako, Osaka, Japan; final concentration of $500 \mu \mathrm{g} / \mathrm{mL}$ ) at $37{ }^{\circ} \mathrm{C}$. Samples prepared from brains were then incubated for $30 \mathrm{~min}$ with proteinase $\mathrm{K}$ (PK; Roche Diagnosis Japan, Tokyo, Japan; final concentration of $40 \mu \mathrm{g} / \mathrm{mL}$ ) at $37^{\circ} \mathrm{C}$. PK digestion was terminated with $2 \mathrm{mM} 4$-(2-aminoethyl) benzenesulfonyl fluoride hydrochloride (Pefabloc; Roche Diagnostics Japan). Samples were mixed with a 2-butanol/methanol mixture (5:1), and PrPres was precipitated by centrifugation at $20000 \times g$ for $10 \mathrm{~min}$ at $20^{\circ} \mathrm{C}$. Pellets were resuspended in Laemmli sample buffer and subjected to WB. Samples were electrophoresed on NuPAGE Novex 12\% Bis-Tris gels with NuPAGE MOPS-SDS running buffer in accordance with the manufacturer's instructions (Life Technologies, Carlsbad, CA, USA). Proteins were then transferred onto Immobilon-P membranes (Millipore, Billerica, MA, USA). The blotted membranes were incubated with the
anti-PrP mAbs listed in Table 1 at $4{ }^{\circ} \mathrm{C}$ overnight. After washing with PBS containing $0.05 \%(\mathrm{v} / \mathrm{v})$ Tween 20 , the membranes were incubated with goat anti-mouse IgGHRP (Jackson ImmunoResearch, West Grove, PA, USA) for $60 \mathrm{~min}$. Signals were developed with a chemiluminescent substrate (SuperSignal; Thermo Fisher Scientific K.K). For semi-quantification, blots were imaged using a Fluorchem system (Alpha Innotech, San Leandro, CA, USA) and analyzed using image reader software (AlphaEaseFC; Alpha Innotech) according to the manufacturer's instructions.

\section{Statistical analysis}

To determine statistical significance the Student's $t$-tests were applied on paired data. Differences with $P<0.05$ were considered significant. Statistical analysis was performed using KaleidaGraph software (Synergy Software, Reading, PA, USA).

\section{Results \\ Characteristics of the $\mathrm{CH} 1641$-like scrapie isolate (Sh294) in TgOvPrP59 mice}

First, we investigated the characteristics of Sh294 in TgOvPrP59 mice. As shown in Figure 1A, CH1641-like scrapie-specific PrPres banding patterns with the typical $\sim 12$-kDa small C-terminal fragment was detected in the brains of TgOvPrP59 mice, which were inoculated with Sh294 by WB analysis using the mAb SAF84. Splenic PrPres was detected in five out of seven TgOvPrP59 mice inoculated with brain homogenates prepared from a sheep showing the classical scrapie PrPres banding pattern (Additional file 1). In contrast, splenic PrPres was not detected in TgOvPrP59 mice infected with Sh294 at any passage number (Figure $1 \mathrm{~B}$ and Table 2). The mean survival period of mice inoculated with Sh294 was $259 \pm 24.5$ at the first passage. Subsequent transmission to TgOvPrP59 mice resulted in a shortened mean survival period ( $215 \pm 17.9$ days at the third passage, Table 2 ).

\section{Biochemical characteristics of PrPres from the $\mathrm{CH} 1641$-like scrapie isolate in TgBoPrP mice}

PrPres banding patterns were compared between TgBo$\operatorname{PrP}$ mice inoculated with the Sh294 isolate and three different BSE strains. The molecular mass of PrPres in the brains of TgBoPrP mice infected with Sh294 was lower than that of TgBoPrP mice infected with $\mathrm{H}$-BSE (Figure 2A). WB analysis using mAb 6H4 demonstrated identical triplet band patterns of PrPres between TgBo$\operatorname{PrP}$ mice infected with Sh294 and those infected with L-BSE (Figure 2A). The mAb SAF84 was used to detect the CH1641-specific $\sim 12-\mathrm{kDa}$ small C-terminal PrPres fragment in sheep affected with the Sh294 scrapie isolate (Figures 2B and D). As previously reported, this antibody 


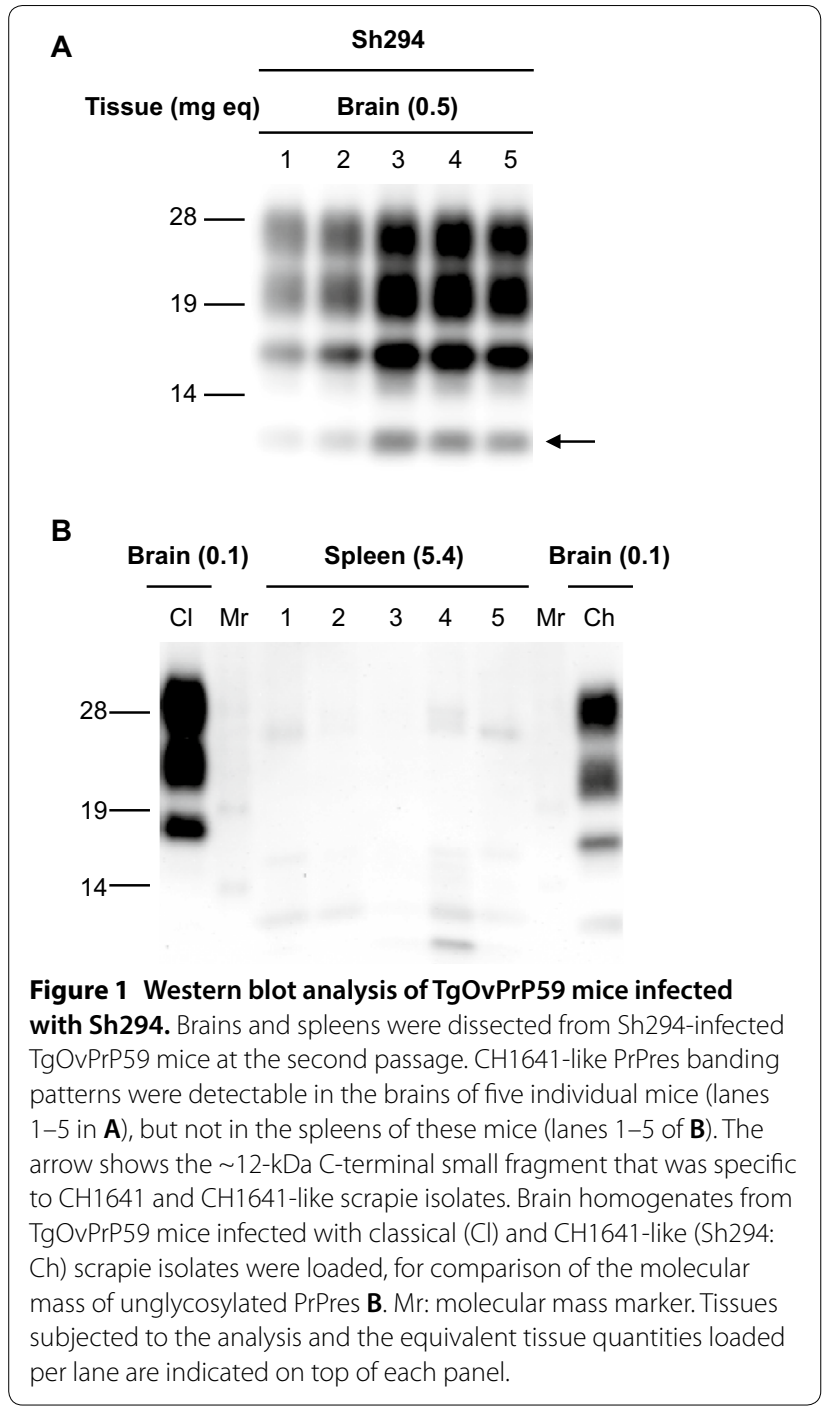

also reacted with a small C-terminal fragment of PrPres in $\mathrm{TgBoPrP}$ mice infected with H-BSE (Figures $2 \mathrm{C}$ and D) $[25,26]$. A similar small PrPres fragment was detected in $\mathrm{TgBoPrP}$ mice infected with Sh294, but not in those infected with L-BSE (Figures $2 \mathrm{~B}$ and D). The mAb P4 reacted only with the PrPres of TgBoPrP mice infected with H-BSE. However, this antibody did not recognize the $\sim 12-\mathrm{kDa}$ small fragment (Figure 2C). Deglycosylation demonstrated that the molecular mass of the PrPres in $\mathrm{TgBoPrP}$ mice infected with Sh294 was slightly lower than that of $\mathrm{TgBoPrP}$ mice infected with C-BSE (lane 1 and 3 in Figure 2D). In contrast, $\mathrm{TgBoPrP}$ mice infected with Sh294 showed nearly the same molecular mass of the PrPres as L-BSE-infected TgBoPrP mice (lanes 1 and 2 in Figure 2D). WB analysis using mAb SAF84 after deglycosylation also revealed that the molecular mass of the small PrPres fragment in TgBoPrP mice infected with Sh294 was slightly higher than that in sheep Sh294 (lanes $\mathrm{Ch}$ and 1 in Figure 2D). Based on the signal intensities measured by the Fluorchem system, the proportions of the $\sim 12-\mathrm{kDa}$ PrPres fragment in TgBoPrP mice infected with Sh294 ( 7\% of total PrPres) were lower than those in sheep Sh294 ( $16 \%$ of total PrPres). As shown in Figure $2 \mathrm{E}$, glycoform profiles of PrPres using the $\mathrm{mAb}$ $6 \mathrm{H} 4$ were significantly different between $\mathrm{TgBoPrP}$ mice infected with C-BSE and Sh294 $(P<0.0001$, Student's $t$-test). However, similar profiles were observed in TgBoPrP mice infected with Sh294 and L-BSE. The results for transmission of the Sh294 isolate to $\mathrm{TgBoPrP}$ mice are given in Table 3. Although TgBoPrP mice infected with Sh294 or L-BSE exhibited unique clinical signs, including circling behavior, the survival period of L-BSE-infected $\operatorname{TgBoPrP}$ mice was significantly shorter than that of TgBoPrP mice infected with Sh294, even after the third passage $(144 \pm 4.8$ days versus $172 \pm 4.5$ days, $P<0.0001$, Student's $t$-test).

\section{Neuropathological comparison between L-BSE and the $\mathrm{CH}$ 1641-like scrapie isolate in TgBoPrP mice} Histological changes and $\operatorname{PrP}^{\mathrm{d}}$ distributions in the brain were compared between $\mathrm{TgBoPrP}$ mice infected with Sh294 and L-BSE (Figure 3). TgBoPrP mice infected with L-BSE exhibited more severe spongiform changes than Sh294-infected TgBoPrP mice, except for changes in the superior cortex, hypothalamus, and the septal nuclei of the paraterminal body (Figures $3 \mathrm{~A}-\mathrm{K}$ ). Neuronal cells in the CA1 area were particularly rarefied in L-BSE-infected $\mathrm{TgBoPrP}$ mice as compared with those in the CA1 area

Table 2 Transmission of Sh294 to TgOvPrP59 mice

\begin{tabular}{|c|c|c|c|c|c|}
\hline \multirow[t]{2}{*}{ Source } & \multirow[t]{2}{*}{ Recipient } & \multirow[t]{2}{*}{ Passage \# } & \multirow[t]{2}{*}{ Survival period $^{a}$} & \multicolumn{2}{|c|}{ PrPres $^{\mathbf{b}}$} \\
\hline & & & & Brain & Spleen \\
\hline \multirow[t]{3}{*}{ Sh294 brain (CH1641-like scrapie) } & TgOvPrP59 & 1 & $259 \pm 24.5$ & $10 / 10$ & $0 / 10$ \\
\hline & & 2 & $233 \pm 13.4$ & $5 / 5$ & $0 / 5$ \\
\hline & & 3 & $215 \pm 17.9$ & $5 / 5$ & $0 / 5$ \\
\hline
\end{tabular}

\footnotetext{
a Survival periods are shown as mean survival days \pm standard deviation.
}

b The number of analyzed mice is shown together with the results of PrPres detection in the brain and spleen (number of PrPres-positive mice/number of inoculated mice). All mice showed clinical signs of disease, such as clasping. 
Figure 2 Biochemical analysis of PrPres from Sh294 in TgBoPrP mice. PrPres banding patterns were compared in TgBoPrP mice infected with Sh294 and BSEs using the mAbs $6 \mathrm{H} 4 \mathbf{A}$ and SAF84 B PrPres was also characterized using the mAbs P4 C and SAF84 D after PNGase deglycosylation. Lane 1, Sh294; lane 2, L-BSE; lane 3, C-BSE; lane 4, H-BSE. Brain homogenates from sheep affected with classical scrapie isolate (lane $\mathrm{Cl}$ in B) and CH1641-like scrapie isolate (Sh294: lane $\mathbf{C h}$ in $\mathbf{B}, \mathbf{D}$ ) were also loaded for comparison. Lanes from 1 to 4 in $\mathbf{A}$, B were loaded with $0.6,0.06,0.5$, and $0.4 \mathrm{mg}$ brain equivalent, respectively. Lanes $\mathrm{Cl}$ and $\mathrm{Ch}$ in $\mathbf{B}$ were loaded with 0.3 and $2.0 \mathrm{mg}$ brain equivalent, respectively. Lanes from 1 to 4 in $\mathbf{C}$ were loaded with $0.03,0.006,0.02$, and $0.02 \mathrm{mg}$ brain equivalent, respectively. Lanes from left to right in $\mathbf{D}$ were loaded with $0.2,0.2,0.06,0.05$ and $0.02 \mathrm{mg}$ brain equivalent, respectively. Molecular markers are shown on the left of each panel. Glycoform profiles of PrPres in brains of TgBoPrP mice infected with Sh294, C-BSE, and L-BSE are given E. PrPres was detected with mAb 6H4. Symbols: red squares, Sh294; blue triangles, C-BSE; green circles, L-BSE.

of Sh294-infected mice (Figures 3B and C). Immunohistochemistry demonstrated the different $\operatorname{PrP}^{\mathrm{d}}$ distribution patterns between $\mathrm{TgBoPrP}$ mice infected with Sh294 and L-BSE. Many granular $\operatorname{PrP}^{\mathrm{d}}$ deposits were detected in the hippocampus of L-BSE-infected $\mathrm{TgBoPrP}$ mice but not Sh294-infected mice (Figures 3L and M).

\section{Interspecies transmission of the $\mathrm{CH} 1641$-like scrapie isolate passaged in TgBoPrP mice to wild-type mice}

To improve the understanding of the differences between Sh294 and L-BSE in TgBoPrP mice, interspecies transmission of TgBo-passaged Sh294 to ICR mice was conducted. Although none of the ICR mice injected with TgBo-passaged Sh294 developed any clinical signs of the disease at the first passage, PrPres was detected by WB analysis in seven of twenty mice sacrificed at the end of their lifespan (Table 4). The mean survival period of mice exhibiting an accumulation of PrPres was $807 \pm 46.9$ days. Subsequent transmission to ICR mice resulted in a shortened mean survival period ( $515 \pm 33.6$ days) with a $100 \%$ infection rate. The third passage in ICR mice further reduced the mean survival period ( $453 \pm 40.4$ days). At the third passage, all inoculated mice accumulated $\operatorname{PrP}^{\mathrm{d}}$ in the brain, and some of them developed clinical signs of the disease, such as rough fur and kyphosis. The sudden death of two out of six inoculated mice occurred at day 477 and 552, respectively, without any clinical signs. However, postmortem analysis revealed that they accumulated $\mathrm{PrP}^{\mathrm{d}}$ in the brain. In contrast, ICR mice infected with sheeppassaged L-BSE still showed a more prolonged survival period compared with that in mice infected with TgBopassaged Sh294 (Table 4). The PrPres banding patterns

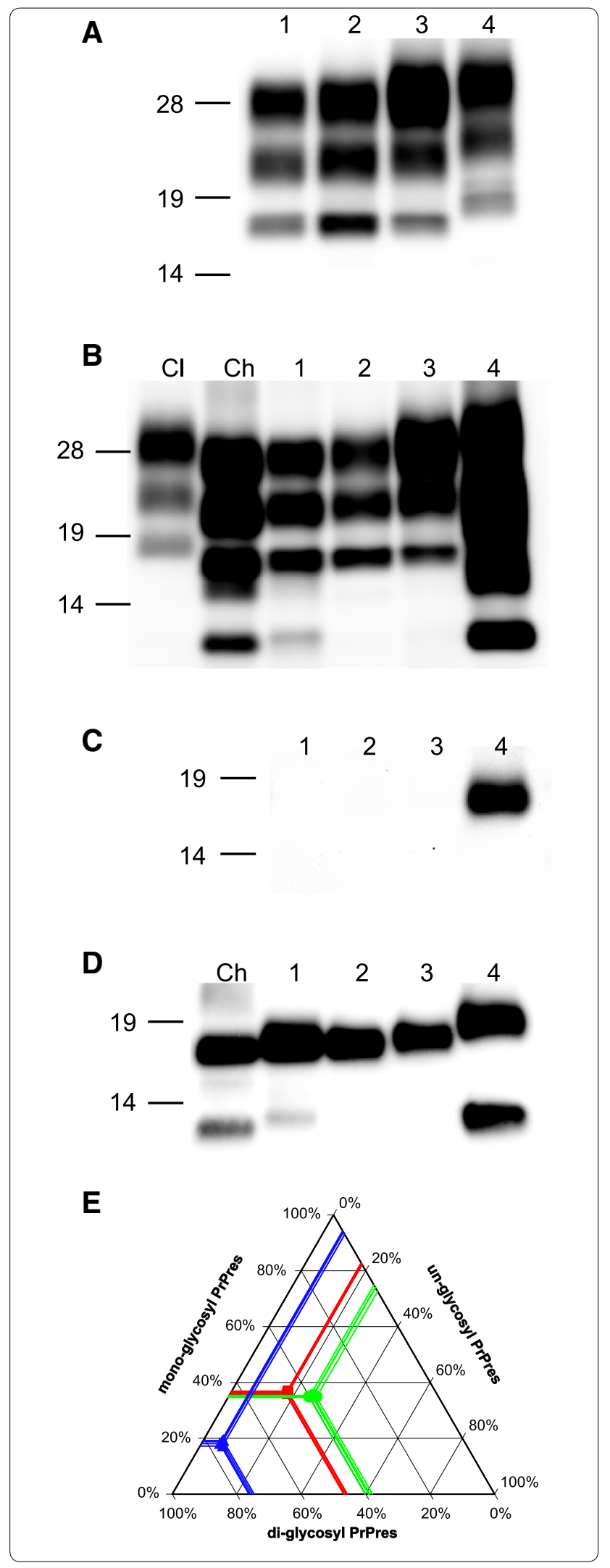


Table 3 Transmission of the $\mathrm{CH} 1641$-like scrapie isolate (Sh294) to TgBoPrP mice

\begin{tabular}{lllll}
\hline Source & Recipient & Passage \# & Survival period $^{\mathbf{a}}$ & $\mathbf{n}^{\mathbf{n}} \mathbf{\mathbf { n } _ { \mathbf { o } }}$ \\
\hline $\begin{array}{l}\text { Sh294 brain } \\
\text { (CH1641-like }\end{array}$ & TgBoPrP & 3 & $172 \pm 4.5^{*}$ & $11 / 11$ \\
$\begin{array}{l}\text { Scrapie) } \\
\text { Cattle L-BSE brain }\end{array}$ & TgBoPrP & 3 & & \\
Cattle C-BSE brain & TgBoPrP & 3 & $144 \pm 4.8^{*}$ & $12 / 12$ \\
Cattle H-BSE brain & TgBoPrP & 3 & $195 \pm 4.6$ & $14 / 14$ \\
\hline
\end{tabular}

a Survival periods are shown as mean survival days \pm standard deviation.

b Number of PrPres-positive mice out of the total number of challenged mice. All inoculated mice showed unique clinical signs of the disease (Sh294 and L-BSE, circling behavior; C-BSE, head twitching; $\mathrm{H}-\mathrm{BSE}$, constant chewing of the bedding).

* $P<0.0001$.

of ICR mice infected with TgBo-passaged Sh294 was different from those of ICR mice infected with sheep-passaged L-BSE (Figures 4A and B). WB analysis using the mAb SAF84 demonstrated reproduction of $\mathrm{CH} 1641$ like scrapie-specific PrPres banding patterns with the $\sim 12-\mathrm{kDa}$ small fragment in ICR mice infected with TgBo-passaged Sh294 (arrow in Figure 4B and lane 3 in Figure $4 \mathrm{C}$ ). This small fragment could not be detected with mAb 6H4 (lane 1-3 in Figure 4A and lane 7 in Figure $4 \mathrm{C}$ ). These results indicate that the small fragment detected in ICR mice infected with TgBo-passaged Sh294 shares similar properties with the C-terminal PrPres fragment detected in sheep Sh294 and TgBoPrP mice infected with Sh294. Deglycosylation demonstrated that the molecular mass of the small C-terminal PrPres fragment was identical between TgBoPrP mice infected with Sh294 and ICR mice infected with TgBopassaged Sh294, but slightly higher than that of the original sheep Sh294 (lane 1-3 in Figure 4C). Glycoform profiles using the mAb $6 \mathrm{H} 4$ demonstrated that the mono-glycosylated PrPres was dominant in the brain of ICR mice infected with TgBo-passaged Sh294 as well as in the brain of ICR mice infected with sheeppassaged L-BSE (Figure 4D). In contrast, the amount of diglycosylated PrPres was dominant in ICR mice infected with mBSE (Figure 4D). Granular $\operatorname{PrP}^{\mathrm{d}}$ deposits were detected in neuronal cells and astrocytes of the hippocampus and thalamus of ICR mice infected with TgBo-passaged Sh294 (Figures 5A and B). Some $\operatorname{PrP}^{\mathrm{d}}$ signals were detected in the cytoplasm of hypertrophied astrocytes (Figure 5C).

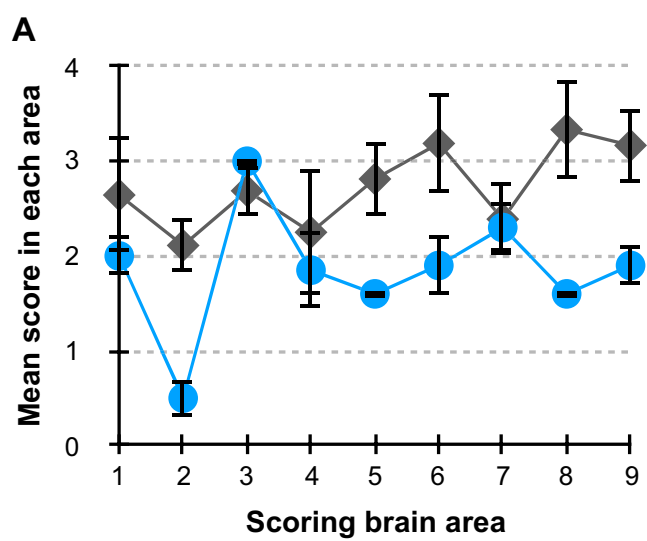

Sh294
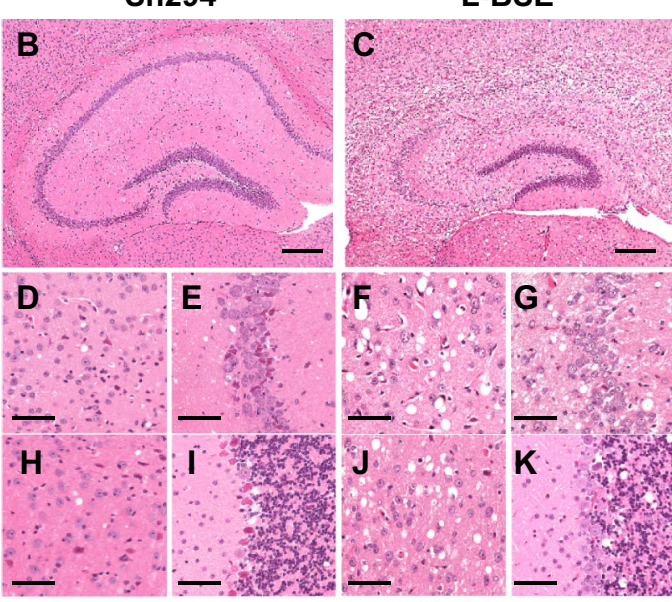

$\mathbf{L}$

M
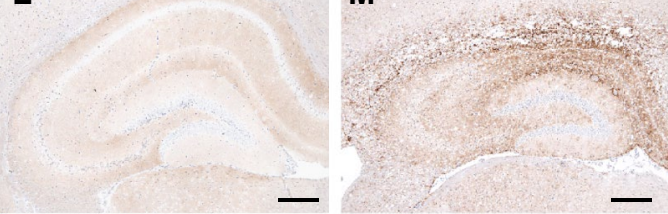

Figure 3 Neuroanatomical changes and $\operatorname{PrP}^{d}$ accumulation in the brains of TgBoPrP mice infected with Sh294 and L-BSE. Vacuolation in the following brain regions was scored on a scale of 0-5 A: 1, dorsal medulla; 2 , cerebellar cortex; 3 , superior cortex; 4 , hypothalamus; 5 , thalamus; 6 , hippocampus; 7 , septal nuclei of the paraterminal body; 8 , cerebral cortex at the level of the hypothalamus and thalamus; 9 , cerebral cortex ash the level of the septal nuclei of the paraterminal body. Results are shown as the mean \pm standard deviation (circles, Sh294; diamonds, L-BSE). Sections were subjected to H\&E staining. Representative examples of vacuolation in the hippocampus B, C, E, G, cortex D, F, thalamus $\mathbf{H}$, J, and cerebellum $\mathbf{I}, \mathbf{K}$ are shown. Representative PrPd immunohistochemistry in the hippocampus is shown $\mathbf{L}, \mathbf{M}$. Scale bars indicate $200 \mu \mathrm{m} \mathbf{B}, \mathbf{C}, \mathbf{L}, \mathbf{M}$ and $50 \mu \mathrm{m} \mathbf{D}-\mathbf{K}$. 


\section{Discussion}

The biological and biochemical properties of the experimental CH1641-like scrapie isolate (Sh294) in TgBoPrP mice were different from those of $\mathrm{H}$ - and C-BSE [25]. In contrast, $\mathrm{TgBoPrP}$ mice infected with Sh294 exhibited clinical signs and the triplet band pattern of PrPres similar to those of mice infected with L-BSE. However, the $\sim 12-\mathrm{kDa}$ small C-terminal fragment of PrPres could only be detected in TgBoPrP mice infected with Sh294. Moreover, the mean survival periods, lesion profiles, and $\operatorname{PrP}^{\mathrm{d}}$ distribution patterns in the brain were distinctly different between the mice infected with Sh294 and those infected with L-BSE. Interspecies transmission of TgBo-passaged Sh294 to wild-type (ICR) mice also demonstrated the biological differences between Sh294 and L-BSE passaged in $\mathrm{TgBoPrP}$ mice. It is known that L-BSE from cattle or TgBoPrP mice is unable to transmit to ICR mice [20, 27]. In contrast, TgBo-passaged Sh294 can transmit to ICR mice even though inefficiently. Since we previously reported that L-BSE could transmit to ICR mice after cross-species transmission to sheep [20], we can now compare the characteristics of the CH1641-like scrapie isolate and L-BSE in wild-type mice. The mean survival period of ICR mice infected with sheep-passaged L-BSE was significantly extended in comparison with that of ICR mice infected with TgBo-passaged Sh294 (Table 4). Although the glycoform profiles were similar between ICR mice infected with TgBo-passaged Sh294 and those infected with sheep-passaged L-BSE, the molecular mass of PrPres was distinctly different between them. In the histopathological analysis, florid plaques were detected in the brains of ICR mice infected with sheep-passaged L-BSE [20], but not in the brains of mice infected with TgBo-passaged Sh294. Thus, all our data demonstrate that the Sh294 isolate is independent of all three BSE strains, suggesting that $\mathrm{CH} 1641$-like scrapie isolates could not be candidates for the origin of BSEs. Indeed, several studies have suggested that spontaneously occurring atypical BSEs in cattle may have been the origin of C-BSE [28-32].

It is of interest to us that TgBo-passaged Sh294 is transmitted to ICR mice with the CH1641-like scrapie-specific PrPres banding patterns because this scrapie isolate cannot transmit directly from sheep to wild-type mice [19]. The biological and biochemical features of TgBo-passaged Sh294 in ICR mice were evidently different from those of sheep-passaged L-BSE. Moreover, these features were also different from all other tested laboratory scrapie strains (22L, ME7, and Chandler), mouse-adapted
Table 4 Transmission of TgBo-passaged Sh294 to wildtype mice

\begin{tabular}{lllll}
\hline Source & Recipient & Passage \# & Survival period $^{\mathbf{a}}$ & $\mathbf{n}^{\mathbf{n}} \mathbf{\mathbf { n } _ { \mathbf { o } }}$ \\
\hline $\begin{array}{c}\text { TgBoPrP mouse } \\
\text { brain infected }\end{array}$ & ICR & 1 & $807 \pm 46.9$ & $7 / 20$ \\
with Sh294 & 2 & $515 \pm 33.6^{*}$ & $6 / 6^{\mathrm{c}}$ \\
Sheep brain & ICR $^{f}$ & 1 & $453 \pm 40.4^{*}$ & $8 / 8^{\mathrm{d}}$ \\
$\begin{array}{l}\text { infected with } \\
\text { L-BSE }\end{array}$ & 2 & $710^{\mathrm{d}}$ & $1 / 15^{\mathrm{e}}$ \\
& 3 & $680 \pm 20.2$ & $7 / 9$ \\
\hline
\end{tabular}

a Survival periods are shown as mean survival days \pm standard deviation.

b Number of PrPres-positive mice out of the total number of challenged mice.

c Two out of six mice were suddenly dead without any clinical signs at $477^{\text {th }}$ and $552^{\text {th }}$ day. The other four mice developed clinical signs of the disease, such as emaciation, rough fur and kyphosis.

d Two out of eight inoculated mice were suddenly dead without any clinical signs at $377^{\text {th }}$ and $441^{\text {th }}$ day, respectively. The other six mice developed the same clinical signs as above.

e The data for the $1^{\text {st }}$ passage obtained from our previous study shown in the reference (20).

f ICR mice infected sheep-passaged L-BSE did not show any clinical even at the $3^{\text {rd }}$ passage.

$* P<0.05$.

Japanese scrapie isolates, and the mBSE strain, which were available in our laboratory (Figures $4 \mathrm{~A}, \mathrm{~B}, \mathrm{D}$, and Additional file 2) [33, 34]. To the best of our knowledge, only a group reported the transmission of French natural CH1641-like scrapie isolates from sheep to wild-type $(\mathrm{C} 57 \mathrm{BL} / 6)$ mice [35]. In contrast to our results, these isolates easily transmitted to C57BL/6 mice. However, the PrPres banding patterns and the histopathological properties observed in diseased mice exhibited a classical scrapie phenotype. This discrepancy could be explained by the co-existence of two different scrapie prions in French natural CH1641-like scrapie isolates, i.e., a classical scrapie prion and a CH1641 scrapie prion. This notion could be supported by the previous finding that some of TgOvPrP4 mice, overexpressing ovine $\mathrm{PrP}_{\mathrm{ARQ}}$, exhibited the classical scrapie PrPres banding patterns after inoculation with the French natural $\mathrm{CH} 1641$-like scrapie isolates $[36,37]$. It has also been reported that TgOvPrP4 mice inoculated with French natural CH1641-like scrapie isolates accumulate PrPres in both the brain and spleen [38]. Although TgOvPrP4 and TgOvPrP59 transgenic mouse lines were derived from different founders, they overexpressed approximately three times as much $\operatorname{PrP}_{\mathrm{ARQ}}$ in the brain under the control of the neuronspecific enolase promoter as that in sheep brains [21]. Since the previous report failed to detect the expression 


\begin{abstract}
Figure 4 Biochemical analysis of PrPres accumulated in the brains of ICR mice infected with TgBo-passaged Sh294. PrPres banding patterns were compared among ICR mice infected with TgBo-passaged Sh294, sheep-passaged L-BSE, mouse-adapted laboratory classical scrapie strains, and mouse-adapted C-BSE using the mAb 6H4 A and SAF84 B. Lane 1, TgBo-passaged Sh294 (first passage in ICR); lane 2, TgBo-passaged Sh294 (second passage in ICR); lane 3, TgBo-passaged Sh294 (third passage in ICR); lane 4, sheep-passaged L-BSE (first passage in ICR); lane 5, sheep-passaged L-BSE (second passage in ICR); lane 6, sheep-passaged L-BSE (third passage in ICR); lane 7, ME7; lane 8, 22L; lane 9, Chandler; lane 10, mouse-adapted C-BSE (mBSE). Laboratory strains (ME7, 22L, Chandler, and $\mathrm{mBSE}$ ) were serially passaged in ICR mice (more than 10 passages). Lanes 1-3, 4 and 5, 6, and 7-10 in A, B were loaded with $0.5 \mathrm{mg}, 2 \mathrm{mg}, 0.1 \mathrm{mg}$, and $0025 \mathrm{mg}$ brain equivalent, respectively. PrPres was also characterized using the mAbs SAF84 (C, lane 1-4) and $6 \mathrm{H} 4$ (C, lane 5-8) after PNGase deglycosylation. 1, sheep Sh294; 2, TgBoPrP mouse infected with Sh294; 3, ICR mouse infected with TgBo-passaged Sh294; 4, TgBoPrP mouse infected with L-BSE. Lanes $1-3$ and 5-7 in $\mathbf{C}$ were loaded with $0.25 \mathrm{mg}$ brain equivalent. Lanes 4 and 8 in $\mathbf{C}$ were loaded with $0.003 \mathrm{mg}$ brain equivalent. The arrow in B shows the $\sim 12-k D a$ C-terminal small fragment. Molecular markers are shown on the left of each panel. Glycoform profiles of PrPres from ICR mice infected with TgBo-passaged Sh294 (third passage), sheep-passaged L-BSE (third passage) and mBSE (14 ${ }^{\text {th }}$ passage) are given $\mathbf{D}$. PrPres was detected with $\mathrm{mAb} 6 \mathrm{H} 4$. Symbols: red squares, mBSE; blue triangles, sheep-passaged L-BSE; green circles, TgBo-passaged Sh294.
\end{abstract}

of ovine PrP mRNA in non-nervous tissues of TgOvPrP4 mice by RT-PCR [39], the precise mechanisms by which $\operatorname{PrP}^{\mathrm{d}}$ accumulates in the spleen of this transgenic mouse line infected with classical scrapie isolates remain unknown. However, considering the ovine $\mathrm{PrP}^{\mathrm{C}}$ expression level and similar response to the classical scrapie isolate, TgOvPrP59 mice may be a useful model to examine the similarity of the Sh294 isolate to French natural CH1641-like isolates. We demonstrated that none of the Sh294 inoculated mice exhibited the PrPres banding patterns of classical scrapie, even after the third passage in TgOvPrP59 mice. Moreover, splenic PrPres was detected in five out of seven TgOvPrP59 mice inoculated with the classical scrapie isolate, but not in any TgOvPrP59 mice inoculated with Sh294 (Additional file 1 and Table 2). Likewise, TgOvPrP4 mice inoculated with the British CH1641 scrapie isolate have been reported not to accumulate PrPres in their spleens [38]. These data strongly support that the experimental Sh294 isolate consisted of a single scrapie prion, similar to the $\mathrm{CH} 1641$ scrapie prion found in the UK. Unlike natural CH1641-like scrapie isolates, selection of a particular scrapie prion might occur through the experimental intraspecies transmission in sheep. Further transmission study of the British $\mathrm{CH} 1641$ scrapie isolate to $\mathrm{TgOvPrP} 59$ mice is needed to

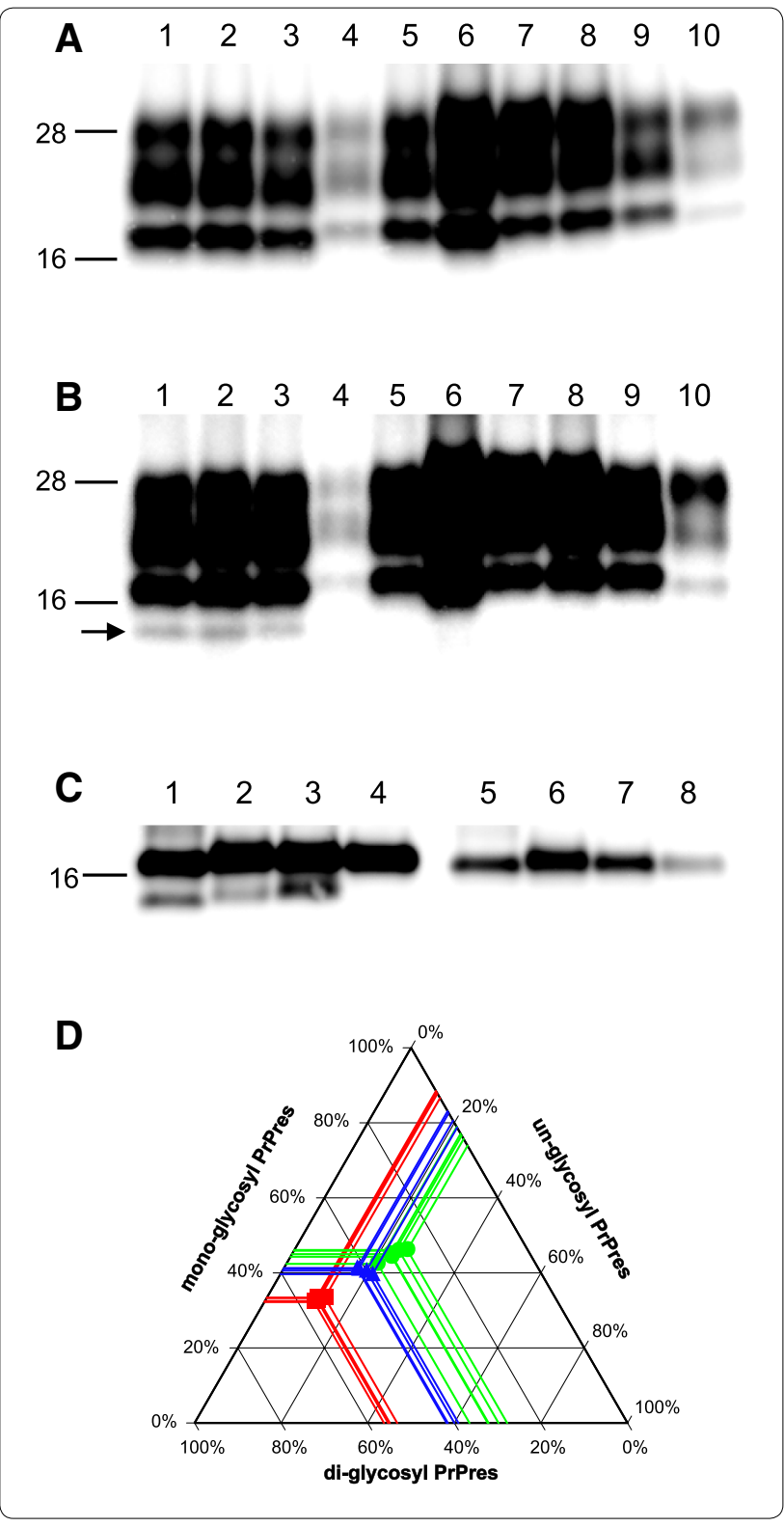

elucidate the similarity between the British CH1641 scrapie isolate and the Sh294 isolate.

The CH1641 scrapie prion remains poorly understood. In this study, we first demonstrated that the CH1641-like scrapie isolate was independent of BSEs, including atypical BSE. Then, we showed that the range of host species susceptible to the $\mathrm{CH} 1641$-like scrapie isolate changed via an interspecies transmission route. Finally, we demonstrated the successful transmission of the CH1641like scrapie isolate (Sh294) to wild-type mice with its specific PrPres banding patterns. Our findings improve 

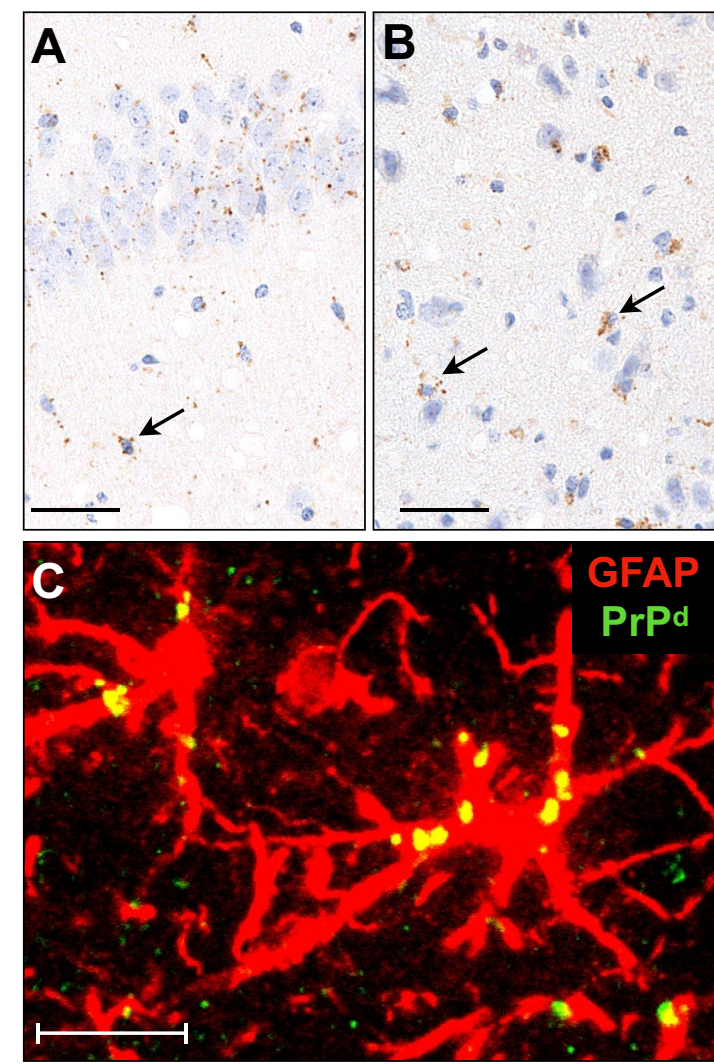

Figure $5 \operatorname{PrP}^{d}$ distribution in the brains of ICR mice infected with TgBo-passaged Sh294. Sections of the hippocampus and thalamus were subjected to immunostaining for $\operatorname{PrP}^{\mathrm{d}} \mathbf{A}-\mathbf{C}$ and GFAP C. Granular PrPd was dispersed in neuronal cells, glial cells (arrows), and neuropils $\mathbf{A}$, B. Some PrPd signals were detected in the cytoplasm of hypertrophied astrocytes (yellow) C. Scale bars indicate $50 \mu \mathrm{m}$ A , B and $10 \mu \mathrm{m} \mathbf{C}$.

the current understanding of the relationship between CH1641-like scrapie isolates and BSEs, including atypical BSE.

\section{Additional files}

\section{Additional file 1. PrPres accumulation in the spleen of TgOvPrP59} mice infected with a classical scrapie isolate. Brains and spleens were dissected from TgOvPrP59 mice inoculated with brain homogenates prepared from a sheep showing the classical scrapie PrPres banding patterns. PrPres was detected with the mAb SAF84. Five out of seven mice (lanes 1-7 of the panels) accumulated the PrPres in the spleen. Brain homogenates from TgOvPrP59 mice infected with classical (Cl) and $\mathrm{CH} 1641$-like (Ch) scrapie isolates were loaded for comparison of the molecular mass of unglycosylated PrPres. Tissues subjected to the analysis and the equivalent tissue quantities loaded per lane are indicated on top of the panel (B).

Additional file 2. Lesion profiles of ICR mice infected with TgBo-passaged Sh294. Vacuolation in the brain regions was scored (A). The brain regions are indicated in Figure 1. Results are shown as the mean \pm standard deviation (circles, TgBo-passaged Sh294 at the third passage; squares, 22L; diamonds, Chandler).

\section{Abbreviations}

BSE: bovine spongiform encephalopathy; PrP: prion protein; PrPd: diseaseassociated PrP; PrPres: protease-resistant core of PrPd; PrPC: cellular PrP; TSE: transmissible spongiform encephalopathy; WB: Western blot; TgBoPrP mice: bovine PrP overexpressing mice; TgOvPrP59: ovine $\operatorname{PrP}_{\mathrm{ARQ}}$ overexpressing mice; $\mathrm{PrP}_{\mathrm{ARQ}}$ : PrP showing the triplet sequences at codons 136, 154 and 171; mBSE: mouse-adapted classical BSE; GFAP: glial fibrillary acidic protein; H\&E: hematoxylin and eosin; mAb: monoclonal antibody.

\section{Competing interests}

The authors declare that they have no competing interests.

\section{Authors' contributions}

$\mathrm{KMi}, \mathrm{KMa}$, $\mathrm{TY}$, and $\mathrm{HO}$ conceived the study. All authors participated in the design of the protocol to conduct the experiments. $\mathrm{KMi}, \mathrm{KMa}$, YM, and $\mathrm{HO}$ performed the experiments. $\mathrm{KMi}, \mathrm{KMa}, \mathrm{TY}$, and $\mathrm{HO}$ analyzed the data. $\mathrm{KMi}$ wrote the manuscript. KMa, YM, YI, TY, and $\mathrm{HO}$ contributed to the revision of the manuscript. All authors read and approved the final manuscript.

\section{Acknowledgements}

We thank Naoko Tabeta, Naomi Furuya, Junko Yamada, Ritsuko Miwa, and the animal caretakers for their expert technical assistance.

\section{Author details}

${ }^{1}$ Prion Disease Unit, Division of Transboundary Animal Disease, National Institute of Animal Health (NIAH), National Agriculture and Food Research Organization (NARO), Tsukuba, Ibaraki, Japan. ${ }^{2}$ Exotic Disease Research Unit, Division of Transboundary Animal Diseases, NIAH, NARO, Kodaira, Tokyo, Japan. ${ }^{3}$ Department of Planning and General Administration, NIAH, NARO, Tsukuba, Ibaraki, Japan.

\section{Ethics approval and consent to participate}

Animal experiments were conducted in strict accordance with the regulations outlined in the Guide for the Care and Use of Laboratory Animals of the National Institute of Animal Health (NIAH) and the Guidelines for Proper Conduct of Animal Experiments, 2006 by the Science Council of Japan. All procedures were approved by the Animal Ethical Committee and the Animal Care and Use Committee of the NIAH (approval ID: 11-008 and 15-005).

\section{Funding}

This work was supported by AMED under Grant Number JP160109044 and partially supported by a research project for improving food safety and animal health of the Ministry of Agriculture, Forestry and Fisheries of Japan.

\section{Publisher's Note}

Springer Nature remains neutral with regard to jurisdictional claims in published maps and institutional affiliations.

Received: 2 July 2018 Accepted: 7 November 2018 Published online: 28 November 2018

\section{References}

1. Collinge J (2001) Prion diseases of humans and animals: their causes and molecular basis. Annu Rev Neurosci 24:519-550

2. Aguzzi A, Calella AM (2009) Prions: protein aggregation and infectious diseases. Physiol Rev 89:1105-1152

3. Soto C (2011) Prion hypothesis: the end of the controversy? Trends Biochem Sci 36:151-158

4. Wells GA, Scott AC, Johnson CT, Gunning RF, Hancock RD, Jeffrey M, Dawson M, Bradley R (1987) A novel progressive spongiform encephalopathy in cattle. Vet Rec 121:419-420

5. Horiuchi M, Nakamitsu S (2007) Prion diseases in animals-bovine spongiform encephalopathy. Nihon Rinsho 65:1513-1520

6. Biacabe AG, Laplanche JL, Ryder S, Baron T (2004) Distinct molecular phenotypes in bovine prion diseases. EMBO Rep 5:110-115

7. Casalone C, Zanusso G, Acutis P, Ferrari S, Capucci L, Tagliavini F, Monaco S, Caramelli M (2004) Identification of a second bovine 
amyloidotic spongiform encephalopathy: molecular similarities with sporadic Creutzfeldt-Jakob disease. Proc Natl Acad Sci U S A 101:3065-3070

8. Fast C, Groschup HM (2013) Classical and atypical scrapie in sheep and goats. In: Zou WQ, Gambetti P (eds) Prions and diseases, animals, humans and the environment, vol 2. Springer, Berlin, pp 15-43

9. Konold T, Nonno R, Spiropoulos J, Chaplin MJ, Stack MJ, Hawkins SA, Cawthraw S, Wilesmith JW, Wells GA, Agrimi U, Di Bari MA, Andreoletti O, Espinosa JC, Aguilar-Calvo P, Torres JM (2015) Further characterisation of transmissible spongiform encephalopathy phenotypes after inoculation of cattle with two temporally separated sources of sheep scrapie from Great Britain. BMC Res Notes 8:312

10. Konold T, Spiropoulos J, Chaplin MJ, Stack MJ, Hawkins SA, Wilesmith JW, Wells GA (2013) Unsuccessful oral transmission of scrapie from British sheep to cattle. Vet Rec 173:118

11. Konold T, Lee YH, Stack MJ, Horrocks C, Green RB, Chaplin M, Simmons MM, Hawkins SA, Lockey R, Spiropoulos J, Wilesmith JW, Wells GA (2006) Different prion disease phenotypes result from inoculation of cattle with two temporally separated sources of sheep scrapie from Great Britain. BMC Vet Res 2:31

12. Clark WW, Hourrigan JL, Hadlow WJ (1995) Encephalopathy in cattle experimentally infected with the scrapie agent. J Infect Dis 56:606-612

13. Cutlip RC, Miller JM, Race RE, Jenny AL, Katz JB, Lehmkuhl HD, DeBey BM, Robinson MM (1994) Intracerebral transmission of scrapie to cattle. J Infect Dis 169:814-820

14. Foster JD, Dickinson AG (1988) The unusual properties of $\mathrm{CH} 1641$, a sheep-passaged isolate of scrapie. Vet Rec 123:5-8

15. Baron T, Bencsik A, Vulin J, Biacabe AG, Morignat E, Verchere J, Betemps D (2008) A C-terminal protease-resistant prion fragment distinguishes ovine "CH1641-like" scrapie from bovine classical and L-Type BSE in ovine transgenic mice. PLoS Pathog 4:e1000137

16. Hope J, Wood SC, Birkett CR, Chong A, Bruce ME, Cairns D, Goldmann W, Hunter N, Bostock CJ (1999) Molecular analysis of ovine prion protein identifies similarities between BSE and an experimental isolate of natural scrapie, CH1641. J Gen Virol 80:1-4

17. Lezmi S, Martin S, Simon S, Comoy E, Bencsik A, Deslys JP, Grassi J, Jeffrey M, Baron T (2004) Comparative molecular analysis of the abnormal prion protein in field scrapie cases and experimental bovine spongiform encephalopathy in sheep by use of Western blotting and immunohistochemical methods. JVirol 78:3654-3662

18. Stack M, Jeffrey M, Gubbins S, Grimmer S, Gonzalez L, Martin S, Chaplin M, Webb P, Simmons M, Spencer Y, Bellerby P, Hope J, Wilesmith J, Matthews D (2006) Monitoring for bovine spongiform encephalopathy in sheep in Great Britain, 1998-2004. J Gen Virol 87:2099-2107

19. Yokoyama T, Masujin K, Schmerr MJ, Shu Y, Okada H, Iwamaru Y, Imamura M, Matsuura Y, Murayama Y, Mohri S (2010) Intraspecies prion transmission results in selection of sheep scrapie strains. PLoS One 5:e15450

20. Okada H, Masujin K, Miyazawa K, Yokoyama T (2015) Acquired transmissibility of sheep-passaged L-type bovine spongiform encephalopathy prion to wild-type mice. Vet Res 46:81

21. Cordier C, Bencsik A, Philippe S, Betemps D, Ronzon F, Calavas D, Crozet C, Baron T (2006) Transmission and characterization of bovine spongiform encephalopathy sources in two ovine transgenic mouse lines (TgOvPrP4 and TgOvPrP59). J Gen Virol 87:3763-3771

22. Matsuura Y, Iwamaru Y, Masujin K, Imamura M, Mohri S, Yokoyama T, Okada H (2013) Distribution of abnormal prion protein in a sheep affected with L-type bovine spongiform encephalopathy. J Comp Pathol 149:113-118

23. Kim CL, Umetani A, Matsui T, Ishiguro N, Shinagawa M, Horiuchi M (2004) Antigenic characterization of an abnormal isoform of prion protein using a new diverse panel of monoclonal antibodies. Virology 320:40-51

24. Shimizu Y, Kaku-Ushiki Y, Iwamaru Y, Muramoto T, Kitamoto T, Yokoyama T, Mohri S, Tagawa Y (2010) A novel anti-prion protein monoclonal antibody and its single-chain fragment variable derivative with ability to inhibit abnormal prion protein accumulation in cultured cells. Microbiol Immunol 54:112-121

25. Okada H, Masujin K, Iwamaru Y, Imamura M, Matsuura Y, Mohri S, Czub S, Yokoyama T (2011) Experimental transmission of H-type bovine spongiform encephalopathy to bovinized transgenic mice. Vet Pathol 48:942-947
26. Masujin K, Okada H, Miyazawa K, Matsuura Y, Imamura M, Iwamaru Y, Murayama Y, Yokoyama T (2016) Emergence of a novel bovine spongiform encephalopathy (BSE) prion from an atypical H-type BSE. Sci Rep $6: 22753$

27. Shu Y, Masujin K, Okada H, Iwamaru Y, Imamura M, Matsuura Y, Mohri S, Yokoyama T (2011) Characterization of Syrian hamster adapted prions derived from L-type and C-type bovine spongiform encephalopathies. Prion 5:103-108

28. Beringue V, Andreoletti O, Le Dur A, Essalmani R, Vilotte JL, Lacroux C, Reine F, Herzog L, Biacabe AG, Baron T, Caramelli M, Casalone C, Laude H (2007) A bovine prion acquires an epidemic bovine spongiform encephalopathy strain-like phenotype on interspecies transmission. J Neurosci 27:6965-6971

29. Baron T, Vulin J, Biacabe AG, Lakhdar L, Verchere J, Torres JM, Bencsik A (2011) Emergence of classical BSE strain properties during serial passages of H-BSE in wild-type mice. PLoS One 6:e15839

30. Capobianco R, Casalone C, Suardi S, Mangieri M, Miccolo C, Limido L, Catania M, Rossi G, Di Fede G, Giaccone G, Bruzzone MG, Minati L, Corona C, Acutis P, Gelmetti D, Lombardi G, Groschup MH, Buschmann A, Zanusso G, Monaco S, Caramelli M, Tagliavini F (2007) Conversion of the BASE prion strain into the BSE strain: the origin of BSE? PLoS Pathog 3:e31

31. Bencsik A, Leboidre M, Debeer S, Aufauvre C, Baron T (2013) Unique properties of the classical bovine spongiform encephalopathy strain and its emergence from $\mathrm{H}$-type bovine spongiform encephalopathy substantiated by VM transmission studies. J Neuropathol Exp Neurol 72:211-218

32. Torres JM, Andreoletti O, Lacroux C, Prieto I, Lorenzo P, Larska M, Baron T, Espinosa JC (2011) Classical bovine spongiform encephalopathy by transmission of H-type prion in homologous prion protein context. Emerg Infect Dis 17:1636-1644

33. Miyazawa K, Okada H, Iwamaru Y, Masujin K, Yokoyama T (2014) Susceptibility of GT1-7 cells to mouse-passaged field scrapie isolates with a long incubation. Prion 8:306-313

34. Masujin K, Shu Y, Okada H, Matsuura Y, Iwamaru Y, Imamura M, Mohri S, Yokoyama T (2009) Isolation of two distinct prion strains from a scrapieaffected sheep. Arch Virol 154:1929-1932

35. Vulin J, Beck KE, Bencsik A, Lakhdar L, Spiropoulos J, Baron T (2012) Selection of distinct strain phenotypes in mice infected by ovine natural scrapie isolates similar to $\mathrm{CH} 1641$ experimental scrapie. J Neuropathol Exp Neurol 71:140-147

36. Baron T, Biacabe AG (2007) Molecular behaviors of "CH1641-like" sheep scrapie isolates in ovine transgenic mice (TgOvPrP4). J Virol 81:7230-7237

37. Arsac JN, Baron T (2014) Distinct transmissibility features of TSE sources derived from ruminant prion diseases by the oral route in a transgenic mouse model (TgOvPrP4) overexpressing the ovine prion protein. PLoS One 9:e96215

38. Baron T, Bencsik A, Morignat E (2010) Prions of ruminants show distinct splenotropisms in an ovine transgenic mouse model. PLoS One 5:e10310

39. Crozet C, Lezmi S, Flamant F, Samarut J, Baron T, Bencsik A (2007) Peripheral circulation of the prion infectious agent in transgenic mice expressing the ovine prion protein gene in neurons only. J Infect Dis 195:997-1006

Ready to submit your research? Choose BMC and benefit from:

- fast, convenient online submission

- thorough peer review by experienced researchers in your field

- rapid publication on acceptance

- support for research data, including large and complex data types

- gold Open Access which fosters wider collaboration and increased citations

- maximum visibility for your research: over $100 \mathrm{M}$ website views per year

At BMC, research is always in progress.

Learn more biomedcentral.com/submissions 